$\Phi=$

\title{
Clinical impacts of the Sudanese anatomical variations in a relation of the recurrent laryngeal nerve to the inferior thyroid artery
}

\author{
Mohamed Elghazali Elhasan ${ }^{1}$, Wael Mohialddin Doush ${ }^{2} *$ \\ ${ }^{1}$ Anatomical Lecturer, MBBS, MSc, Almadain College of Medical Sciences and Technology, Khartoum, Sudan \\ ${ }^{2}$ Assistant Professor of General Surgery, MBBS, MSc, MD, MRCSEd, Faculty of Medicine and Health Sciences, Omdurman Islamic Uni- \\ versity, Department of Gastroenterological Surgery, Ibn Sina Specialized Hospital, Khartoum, Sudan \\ *Corresponding author: Dr.Wael Mohialddin Ahmed Doush, MD, MRCSEd, Assistant Professor of General Surgery, Faculty of Medi- \\ cine and Health Sciences, Omdurman Islamic University, Department of Gastroenterological Surgery, Ibn Sina Specialized Hospital, \\ P.O.Box 7597, Khartoum 11123, Sudan.Mobile phone: +249124529752; E-mail:dr.wael.doush@gmail.com
}

\begin{abstract}
Background: Until recently, the knowledge of the anatomical variations in the relationship between the recurrent laryngeal nerve (RLN) and the inferior thyroid artery (ITA) combined with visual intraoperative RLN identification are essential for the protection of these lifeimportant structures during surgical exposure and the mobilization of thyroid and parathyroid glands. This leads to the proper treatment of patients and improvement of the surgical techniques.

Aim: To describe anatomical variations in the relationship of the recurrent laryngeal nerve to the inferior thyroid artery in the Sudanese population.

Patients and methods: Following ethical committee approval, an observational descriptive prospective cross-sectional study in the period between March 2019 and February 2020, for anatomical variations in the relation of the recurrent laryngeal nerve to the inferior thyroid artery in seventy-two cervical sides of thirty-six well-dissected embalmed Sudanese cadavers. The RLN was investigated in three positions: posterior to the ITA main trunk, anterior to the ITA main trunk and between the ITA branches. Then, the results were analyzed according to the gender, age and dissection side.

Results: We found that the anatomical variations were more frequent in males (97.2\%) than females (2.8\%). The age of these variations ranged between $31-45$ years which represents $(61.1 \%)$. On the right side of the neck, the commonest position of the RLN is posterior to the ITA (63.9\%) followed by in decreasing order of frequency, the RLNs run between the ITA branches (30.6\%) and anterior to the ITA $(5.6 \%)$. On the left side of the neck, the commonest position of the RLN is posterior to the ITA $(69.4 \%)$. It passed between the ITA branches in (30.6\%) of specimens. There was no anterior location of the RLN to the ITA. Variations in a relationship of the RLN to the ITA on one side is significantly different from the opposite side.

Conclusions: Although the risk of potential damage to the RLN during surgical neck procedures involving the thyroid gland and parathyroid glands is well recognized, pre-operative detailed surgeon's knowledge for these frequent anatomical variations and the usage of the ITA as an anatomical landmark for intraoperative RLN recognition is important. This will lead to a reduction of iatrogenic RLN injury prevalence. Future studies are recommended to compare the findings on this cadaveric study with a larger sample size in the long-term period.
\end{abstract}

Keywords: Anatomical Variations; Iatrogenic Injury; Inferior Thyroid Artery; Recurrent Laryngeal Nerve; Sudan; Thyroid Gland.

\section{Introduction}

In 1927, Charles Higgins has published a study including this statement: "the prevention of injuries of the recurrent laryngeal nerve demands an accurate understanding of the anatomical relations of the nerve on the part of everyone who deals with surgery of the larynx or the thyroid gland. The fact that anomalies of the nerve may occur must be constantly kept in mind. The patient who has suffered an injury to the recurrent nerve must always be kept under observation in order to prevent an unfortunate sequel" (Higgins 1927). Until recently, the knowledge of the anatomical variations in the relationship between the recurrent laryngeal nerve (RLN) and the inferior thyroid artery (ITA) combined with visual intraoperative RLN identification are essential for the protection of these life-important structures during surgical exposure and the mobilization of thyroid and parathyroid glands. This leads to the proper treatment of patients and improvement of the surgical techniques (Rimi and Bose 2010; Premachandra et al. 1990). Moreover, RLN injury during ITA ligation is related to less anatomical knowledge. This complication ranges from hoarseness of voice in unilateral injury to acute airway obstruction and stridor in bilateral lesions (Erbil et al. 2007; Jeannon et al. 2009). These serious morbidities despite the modern surgical advances occurred in 14\% of thyroidectomies particularly in total lobectomy for giant nodular goiter and locally advanced thyroid tumours. Also, other studies showed that the surgical iatrogenic injuries to the RLN is one of the most common causes of vocal fold paralysis (37\% of cases) (Tang et 
al. 2012; Kaisha et al. 2011; Gremillion et al. 2012). This is due to poor intraoperative surgical skills listed by (Dionigi et al. 2016) as follows: traction $(71 \%)$, electro-thermal cautery to stop vascular bleeding $(17 \%)$, compression $(4.2 \%)$, vascular clamping $(3.4 \%)$, ligature entrapment (1.6\%), suction (1.4\%) and nerve transection (1.4\%). Furthermore, Zuckerkandl's tuberculum is defined as a lateral extension of the thyroid lobes behind the trachea. It increases the difficulty of intraoperative RLN localization (Cannizzaro 2004).

\subsection{Clinical anatomy of the RLN and ITA}

The recurrent laryngeal nerve (RLN) has integrating functions in the phonation, airways patency and swallowing. It has been described as a mixed branch of the vagus nerve. At the root of the neck, the right RLN hooking around the right subclavian artery, while in the thorax the left RLN hooking around the ligamentum arteriosum. In the neck, both nerves passed along the tracheoesophageal groove to enter the posterior surface of the larynx at the level of cricoid cartilage behind the cricothyroid muscle and become proximal to the posteromedial aspect of the thyroid gland. This area is called Killian's area where the RLN is frequently damaged (Akil et al. 2017; Sasaki and Mitra 2001). The inferior thyroid artery (ITA) originates from the thyrocervical trunk which a branch of the first part of the subclavian artery. Then, behind the carotid sheath, the ITA ascends vertically, curving medially and posteriorly to reach the tracheoesophageal groove on the anterior surface of the longus coli muscle. After that, it penetrates the prevertebral fascia to cross over the RLN and divides into an ascending branch which supplies the posterior surface of the thyroid gland and the descending branch to supply the inferior parathyroid gland and lower pole of the thyroid gland (Arrangoiz et al. 2018).

\subsection{Patterns of anatomical variations in a relation of the recurrent laryngeal nerve to the inferior thyroid artery}

The anatomical relationship of the RLN to the ITA is highly variable in the literature. (Rimi and Bose 2010) had classified these anatomical variations into three important types. The RLN either located posterior, anterior or it may pass through the ITA terminal branches in 40\%, 20\% and 35\% of specimens respectively (Figure 1), (Akil et al. 2017; Arrangoiz et al. 2018; Zada et al. 2014).
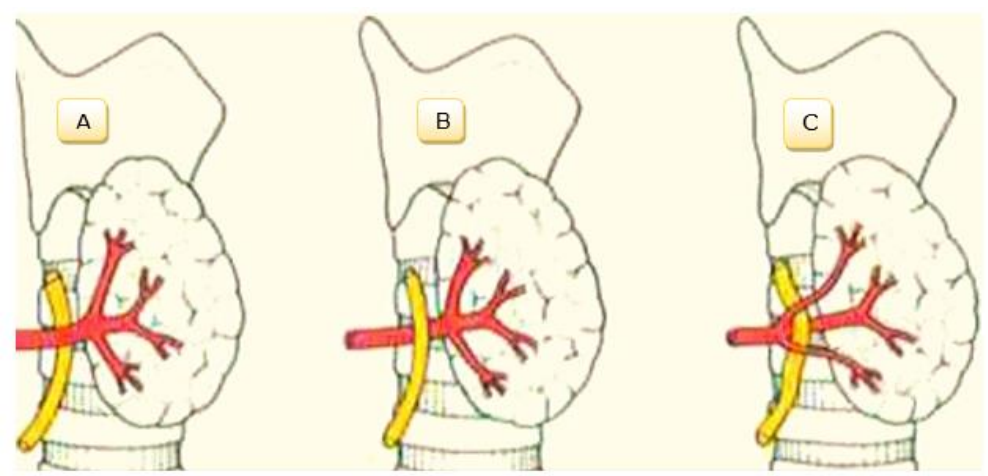

Fig. 1: The Recurrent Laryngeal Nerve Courses with the Inferior Thyroid Artery Including Posterior to the ITA Trunk (A), Anterior to the ITA Trunk (B) and Passed between the ITA Branches (C).

The extralaryngeal terminal bifurcation (ETB) of RLN is an anatomical variation defined as division of the RLN into an anterior and posterior branch near the level of Berry's ligament which anchors the thyroid gland to the upper three tracheal cartilage rings. It was reported in $30 \%$ of specimens and may occur bilaterally in $25 \%$ of specimens. Katz \& Nemiroff were reported this variation in $63.9 \%$ of cases (Katz and Nemiroff 1993). The sites of bifurcation point related to ITA either prearterial, arterial and postarterial in $11 \%, 39 \%$ and $50 \%$ of cases respectively (Cetin et al. 2016; Nyeki et al. 2015; Sasou et al. 1998). The iatrogenic injury rate reported as 5.2\% and 1.6\% for bifid and non-bifid nerves respectively. This was related to visual misidentification because of partial intraoperative dissection of RLN main trunk and its motor fibres. Moreover, the serious complications are more in anterior branch damage rather than posterior branch due to it containing high motor fibres (Cetin et al. 2016). A rare anatomical variation of the RLN is non-RLN which is direct origin of the nerve from the vagus nerve without the usual recurrent course. This is mostly seen on the right side of the neck reported in $0.3-1.6 \%$. It is classified into two types: the first, non-RLN mistaken as a branch of the superior thyroid artery and the second when nonRLN is considered as a branch of ITA (Konschake et al. 2016). Stedman was the first author who discovered this variation in 1823 (Sanders et al. 1983). Moreover, Randolph had revealed another variation known as a distorted RLN which result from large substernal goiter that displaced the RLN to the ventral side of the lower thyroid pole or it is adherent to the capsule of large recurrent goiter which is injured during lateral dissection of the goiter (Randolph 2003).

Furthermore, in $62.68 \%$ of persons when these variations exist on one side they will not be repeated on the opposite side of the same individuals. (Ozguner and Sulak 2014) distinguished different positions of the RLN to the ITA as follows: RLN located posterior to the ITA: right (42.5\%); left (65\%), RLN located anterior to the ITA: right (40.5\%); left (22.5\%) and RLN pass between the ITA branches: right $(1 \%)$; left $(3.5 \%)$.

\subsection{Aims and objectives}

To describe anatomical variations in the relationship of the recurrent laryngeal nerve to the inferior thyroid artery in the Sudanese population. There are no cadaveric-based studies of these frequent variations in Sudan and few publications specifically described this anomaly.

\section{Materials and methods}

The study received no grant funding and was held in the dissecting rooms of the medical colleges at Khartoum, capital of Sudan. Ethical approval for the Sudanese cadaveric dissection was obtained from the local Research Ethics Committee of Faculty of Medicine and Health Sciences, Omdurman Islamic University before the study commenced. This is an observational descriptive prospective cross- 
sectional study in the period between March 2019 and February 2020, for anatomical variations of the RLN positions to the ITA which is divided into posterior to the ITA main trunk, anterior to the ITA main trunk and between the ITA branches. During this period, eightythree Sudanese cadavers (79 males and 4 females) were dissected. The exclusion criteria included the following: diseased cadavers, bad cadaveric storage and careless destructive dissection to the cadaveric cervical region. Hence, seventy-two cervical sides of thirty-six well-dissected embalmed cadavers (35 males and 1 female) with intact anatomy of the RLN and the ITA preserved in $10 \%$ formalin solution were included within this study. Then, the results were analyzed according to the gender, age and dissection side.

\subsection{The RLN dissection technique}

The dissection starts with a longitudinal skin incision in the midline of the neck with two curved transverse incisions, giving two cylindrical cutaneous flaps which are retracted laterally with sternocleidomastoid muscle leading to easy cervical access. Then, the anterior triangle of the neck was carefully dissected through sub-platysmal flaps with the removal of surrounding subcutaneous adipose tissue and muscles reaching the visible thyroid gland to observe cervical courses of both RLN main trunk and ITA from the tracheoesophageal groove to the lower pole of the thyroid gland after medial mobilization of its bilateral lobes.

\subsection{Statistical analysis}

The data were documented in a master sheet and then analyzed by authors using the computer program called Statistical Package for Social Sciences (SPSS) version 24 and excel 2013 for Windows software. Finally, the differences in our results were considered statistically significant if $\mathrm{P}$-value $<0.05$.

\section{Results}

\subsection{Demographic data}

In this study, the analysis of seventy-two cervical sides of thirty-six well-dissected embalmed Sudanese cadavers regarding the gender, age as follows: $35(97.2 \%)$ were males and there is one female representing (2.8\%), (Figure 2). The peak incidence of the specimen's age for these variations ranged between $31-45$ years, which represents (61.1\%), (Figure 3).

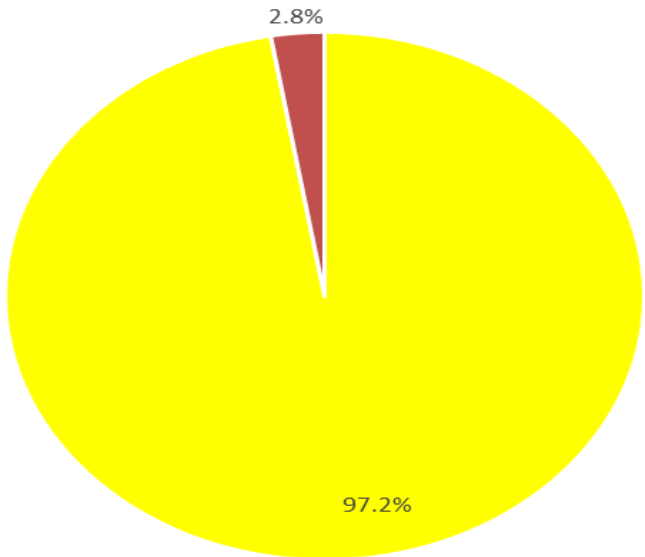

- Female

- Male

Fig. 2: The Gender Distribution.

\subsection{Anatomical variations in a relation of the recurrent laryngeal nerve to the inferior thyroid artery regarding the dissection side}

On the right side, the RLN passes posterior to the ITA in 23 cadavers (63.9\%), between the ITA branches in 11 cadavers (30.6\%) and anterior to the ITA in 2 cadavers $(5.6 \%)$. The difference was significant statistically ( $\mathrm{P}<0.05)$, (Figure 4$)$. On the left side, the RLN was located posteriorly in 25 cadavers $(69.4 \%)$, between the ITA branches in 11 cadavers $(30.6 \%)$ and anterior location to the ITA not observed in this side, (Figure 5). The difference was not significant statistically ( $\mathrm{P}>0.05)$. Variations in relationships of the RLN to the ITA on one side is significantly different from the opposite side. Other forms of congenital anomalies were not found in this study. 


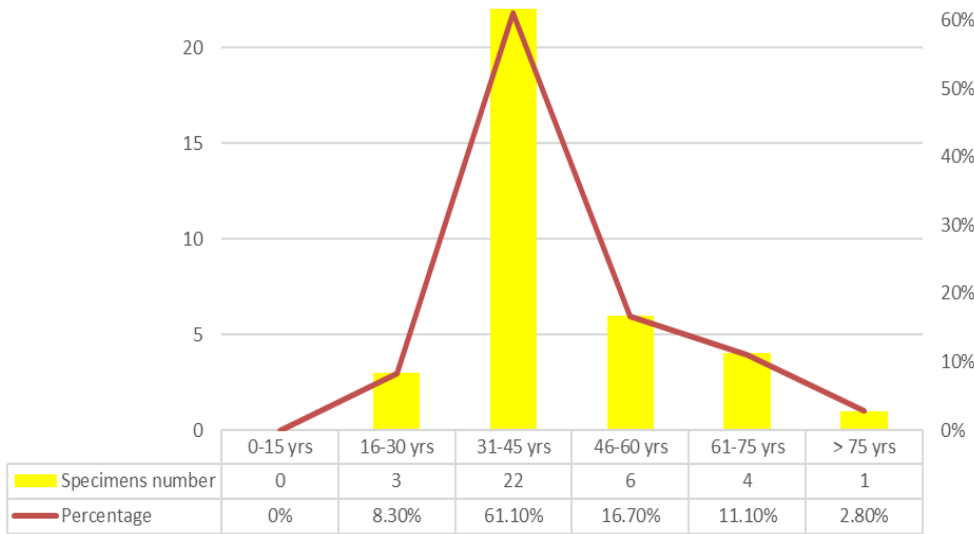

Fig. 3: Age Distribution.

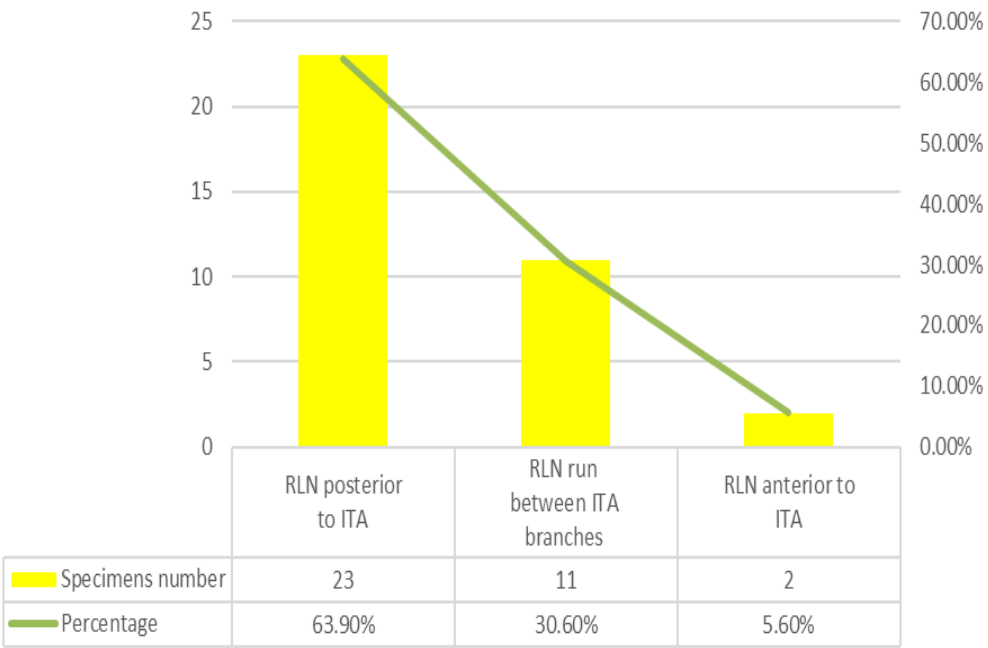

Fig. 4: Anatomical Variations in the Relation of RLN to ITA Regarding the Right Neck Side.

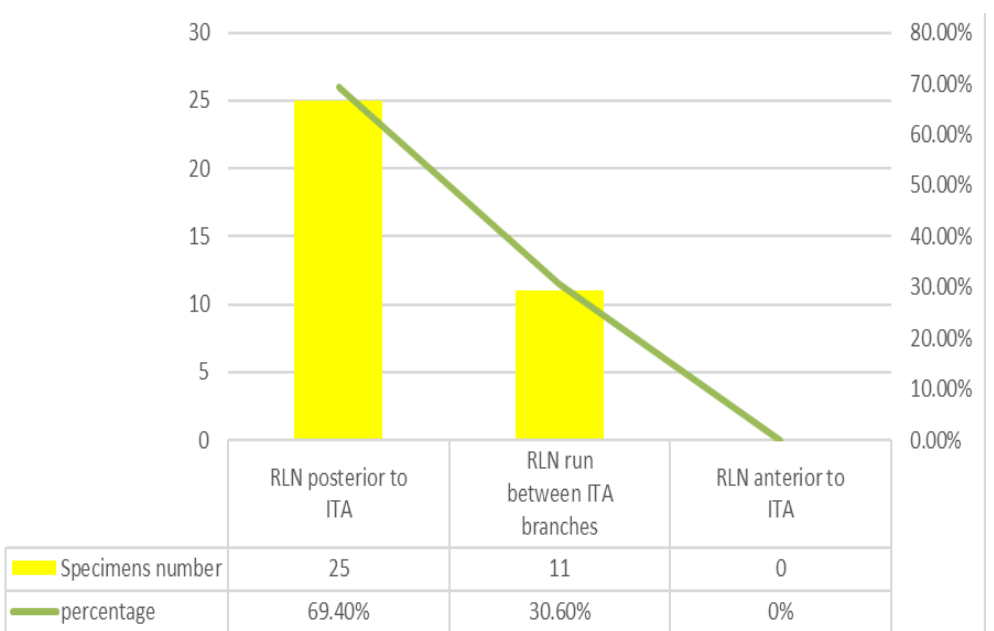

Fig. 5: Anatomical Variations in the Relation of RLN to ITA Regarding the Left Neck Side.

\section{Discussion}

Due to a lack of the Sudanese cadaveric-based studies, this study focuses on the presence of anatomical variations in the relation of the RLN to the ITA regarding both sides of the neck in Sudanese. Many of anatomists and surgeons studied the entire course of the RLN near the thyroid gland using the ITA as an anatomical landmark when performing surgical procedures in the anterior triangle of the neck such as thyroidectomy, para-thyroidectomy, Zenker's diverticulum excision, esophagectomy and tracheostomy. Hence, full knowledge to identify variable relations of the RLN is essential for prevention of the iatrogenic injury (Premachandra et al. 1990; Campos and Henriques 2000; Dralle et al. 2004).

$97.2 \%$ of the variations in this study were males and $2.8 \%$ were females. These findings are in keeping with those found by (Hirata 1992) in his cadaveric study (40\% vs. $28.6 \%$ ). Moreover, the paucity of female cadavers in this study explained by Sudanese social traditions that don't favour the usage of female cadavers among the medical colleges.

The peak incidence of age in these variations ranged between 31-45 years which represents $61.1 \%$. Extensive revision of published studies in the literature was done. No findings described the importance of cadaveric age in discovery of variable positions of the RLN. 
On the right side of the neck, the RLN passes posterior to the ITA in $63.9 \%$ of specimens, the RLN runs between the ITA branches in $30.6 \%$ of specimens and it passes anterior to the ITA in 5.6\% of specimens. These results are similar to (Roshan et al. 2017) who reported the RLN passage posterior to the ITA in $66 \%$, between the ITA branches in $34 \%$ and absence of RLN anterior to the ITA. Also, other studies (Hirata 1992; Lekacos et al. 1992; Kulekci et al. 2012) revealed the RLN predominantly located posterior to the ITA.

On the left side of the neck, the RLN is located posterior to the ITA in $69.4 \%$ of specimens, the passage of the RLN between the ITA branches was present in $30.6 \%$ of specimens and no anterior location of the left RLN to the ITA. These findings are similar to (Roshan et al. 2017) that showed the RLN running posterior to the ITA in $74 \%$, between the ITA branches in $26 \%$ and no anterior relation to the ITA. (Rimi and Bose 2010) had similar results in Bangladesh revealing the RLN most frequently located posterior to the ITA and least to be anterior in the left side. Moreover, these findings in this study are similar to (Page et al. 2003; Polednak 2006; Beneragama and Serpell 2006; Shao et al. 2010 ) who studied a lot of thyroidectomy cases and reported the more frequent variation on the left side was the passage of the RLN posterior to the ITA. Furthermore, during withdrawal of the thyroid gland from its bed in a total thyroidectomy, the RLN is more prone to be injured when located anteriorly to the ITA or running between its branches than when it lies posterior to the ITA. This will make the surgeons more alert to place the ligature in the ITA away from lower pole of the thyroid gland to safeguard the RLN (Skandalakis et al. 1976; Bhargav 2014).

Eventually, the patterns of anatomical variations on one side of the neck are different in the orientation than the opposite side among the cadavers in this current study. This is similar to the findings by (Reed 1943) in his cadaveric study. Moreover, these results are greater than the findings of (Ozguner and Sulak 2014).

\section{Conclusions}

Although the risk of potential damage to the RLN during surgical neck procedures involving the thyroid gland and parathyroid glands is well recognized, pre-operative detailed surgeon's knowledge for these frequent anatomical variations and the usage of the ITA as an anatomical landmark for intraoperative RLN recognition is important. This will lead to a reduction of iatrogenic RLN injury prevalence. Future studies are recommended to compare the findings on this cadaveric study with a larger sample size in the long-term period.

\section{Conflict of interest}

The authors declare that they have no conflict of interest.

\section{Author contributions}

WMD is responsible for original manuscript writing, editing, supervision and critical revision of contents. WMD and MEE are responsible for data collection, data analysis and manuscript design. WMD and MEE are responsible for manuscript drafting and revision. All authors read and gave the final approval of the manuscript to be published.

\section{Funding information}

There is no financial support and sponsorship from any institute.

\section{Ethics approval and consent to participate}

This original article has been approved ethically by our institution.

\section{Acknowledgments}

The authors really would like to thank their colleagues in the anatomical and surgical departments for their kind suggestions and unlimited support.

\section{ORCID}

Dr. Wael Doush https://orcid. org/0000-0002-4099-5255.

\section{References}

[1] Higgins, C.C., 1927. Surgical anatomy of the recurrent laryngeal nerve with especial reference to thyroid surgery. Annals of surgery, 85(6), p.827838. https://doi.org/10.1097/00000658-192706000-00004.

[2] Rimi, K.R. and Bose, B.K., 2010. Position of terminal branches of inferior thyroid arteries in relation to recurrent laryngeal nerve. Dinajpur Med Col J, 3(2), pp.47-54.

[3] Premachandra, D.J., Radcliffe, G.J. and Stearns, M.P., 1990. Intraoperative identification of the recurrent laryngeal nerve and demonstration of its function. The laryngoscope, 100(1), pp.94-96. https://doi.org/10.1288/00005537-199001000-00019.

[4] Erbil, Y., Barbaros, U., Işsever, H., Borucu, I., Salmaslıoglu, A., Mete, O., Bozbora, A. and Ozarmagan, S., 2007. Predictive factors for recurrent laryngeal nerve palsy and hypoparathyroidism after thyroid surgery. Clinical otolaryngology, 32(1), pp.32-37. https://doi.org/10.1111/j.13652273.2007.01383.x.

[5] Jeannon, J.P., Orabi, A.A., Bruch, G.A., Abdalsalam, H.A. and Simo, R., 2009. Diagnosis of recurrent laryngeal nerve palsy after thyroidectomy: a systematic review. International journal of clinical practice, 63(4), pp.624-629. https://doi.org/10.1111/j.1742-1241.2008.01875.x.

[6] Tang, W.J., Sun, S.Q., Wang, X.L., Sun, Y.X. and Huang, H.X., 2012. An applied anatomical study on the recurrent laryngeal nerve and inferior thyroid artery. Surgical and radiologic anatomy, 34(4), pp.3254-332. https://doi.org/10.1007/s00276-011-0905-8. 
[7] Kaisha, W., Wobenjo, A. and Saidi, H., 2011. Topography of the recurrent laryngeal nerve in relation to the thyroid artery, Zuckerkandl tubercle, and Berry ligament in Kenyans. Clinical anatomy, 24(7), pp.853-857. https://doi.org/10.1002/ca.21192.

[8] Gremillion, G., Fatakia, A., Dornelles, A. and Amedee, R.G., 2012. Intraoperative recurrent laryngeal nerve monitoring in thyroid surgery: is it worth the cost?. Ochsner Journal, 12(4), pp.363-366.

[9] Dionigi, G., Wu, C.W., Kim, H.Y., Rausei, S., Boni, L. and Chiang, F.Y., 2016. Severity of recurrent laryngeal nerve injuries in thyroid surgery. World journal of surgery, 40(6), pp.1373-1381. https://doi.org/10.1007/s00268-016-3415-3.

[10] Cannizzaro, M.A., 2004. Zuckerkandl's tuberculum: could it be useful in thyroid surgery? Chir Ital, 56(5), pp.611-615.

[11] Akil, F., Yollu, U., Ayral, M., Turgut, F. and Yener, M., 2017. The anatomical relationship between recurrent laryngeal nerve and first tracheal ring in males and females. Clinical and experimental otorhinolaryngology, 10(1), p.104-108. https://doi.org/10.21053/ceo.2015.01746.

[12] Sasaki, C.T. and Mitra, S., 2001. Recurrent laryngeal nerve monitoring by cricopharyngeus contraction. The laryngoscope, 111(4), pp.738-739. https://doi.org/10.1097/00005537-200104000-00032.

[13] Arrangoiz, R., Cordera, F., Caba, D., Muñoz, M., Moreno, E. and de Leon, E.L., 2018. Comprehensive review of thyroid embryology, anatomy, histology, and physiology for surgeons. International Journal of otolaryngology and head \& neck surgery, 7(4), pp.160-188. https://doi.org/10.4236/ijohns.2018.74019.

[14] Zada, B., Anwar, K., Malik, S.A., Khan, N. and Salam, F., 2014. Anatomical relationship between recurrent laryngeal nerve and inferior thyroid artery in thyroidectomy patients. Journal of ayub medical college abbottabad, 26(3), pp.380-383.

[15] Katz, A.D. and Nemiroff, P., 1993. Anastamoses and bifurcations of the recurrent laryngeal nerve--report of 1177 nerves visualized. The American surgeon, 59(3), pp.188-191.

[16] Cetin, F., Gurleyik, E. and Dogan, S., 2016. Morphology and functional anatomy of the recurrent laryngeal nerve with extralaryngeal terminal bifurcation. Anatomy research international, 2016, pp.1-5. https://doi.org/10.1155/2016/9503170.

[17] Nyeki, A.R.N., Njock, L.R., Miloundja, J., Vokwely, J.E.E. and Bengono, G., 2015. Recurrent laryngeal nerve landmarks during thyroidectomy. European annals of otorhinolaryngology, head and neck diseases, 132(5), pp.265-269. https://doi.org/10.1016/j.anorl.2015.08.002.

[18] Sasou, S., Nakamura, S.I. and Kurihara, H., 1998. Suspensory ligament of Berry: its relationship to recurrent laryngeal nerve and anatomic examination of 24 autopsies. Head \& neck: journal for the sciences and specialties of the head and neck, 20(8), pp.695-698. https://doi.org/10.1002/(SICI)1097-0347(199812)20:8<695::AID-HED6>3.0.CO;2-3.

[19] Konschake, M., Zwierzina, M.E., Pechriggl, E.J., Moriggl, B., Brenner, E., Hormann, R. and Prommegger, R., 2016. The nonrecurrent laryngeal nerve: a clinical anatomic mapping with regard to intraoperative neuromonitoring. Surgery, 160(1), pp.161-168. https://doi.org/10.1016/j.surg.2015.12.021.

[20] Sanders, G., Uyeda, R.Y. and Karlan, M.S., 1983. Nonrecurrent inferior laryngeal nerves and their association with a recurrent branch. The American journal of surgery, 146(4), pp.501-503. https://doi.org/10.1016/0002-9610(83)90240-4.

[21] Randolph, G.W., 2003. Surgical anatomy of the recurrent laryngeal nerve. Surgery of the thyroid and parathyroid glands, pp.300-302.

[22] Ozguner, G. and Sulak, O., 2014. Arterial supply to the thyroid gland and the relationship between the recurrent laryngeal nerve and the inferior thyroid artery in human fetal cadavers. Clinical anatomy, 27(8), pp.1185-1192. https://doi.org/10.1002/ca.22448.

[23] Campos, B.A. and Henriques, P.R.F., 2000. Relationship between the recurrent laryngeal nerve and the inferior thyroid artery: a study in corpses. Revista do Hospital das Clínicas, 55(6), pp.195-200. https://doi.org/10.1590/S0041-87812000000600001.

[24] Dralle, H., Sekulla, C., Haerting, J., Timmermann, W., Neumann, H.J., Kruse, E., Grond, S., Mühlig, H.P., Richter, C., Voß, J. and Thomusch, O., 2004. Risk factors of paralysis and functional outcome after recurrent laryngeal nerve monitoring in thyroid surgery. Surgery, 136(6), pp.1310 1322. https://doi.org/10.1016/j.surg.2004.07.018.

[25] Hirata, K., 1992. Relationship between the recurrent laryngeal nerve and the inferior thyroid artery in Japanese. Kaibogaku zasshi. Journal of anatomy, 67(5), pp.634-641.

[26] Roshan, S., Bhivate, V.R., Pandey, N. and Shinde, S.B., 2017. Study on recurrent laryngeal nerve: its position and relationship with inferior thyroid artery. Int J Anat Res, 5(1), pp.3404-3409. https://doi.org/10.16965/ijar.2016.490.

[27] Lekacos, N.L., Tzardis, P.J., Sfikakis, P.G., Patoulis, S.D. and Restos, S.D., 1992. Course of the recurrent laryngeal nerve relative to the inferior thyroid artery and the suspensory ligament of Berry. International surgery, 77(4), p.287-288.

[28] Kulekci, M., Batıoglu-Karaaltın, A., Saatci, O. and Uzun, I., 2012. Relationship between the branches of the recurrent laryngeal nerve and the inferior thyroid artery. Annals of otology, rhinology \& laryngology, 121(10), pp.650-656. https://doi.org/10.1177/000348941212101005.

[29] Page, C., Foulon, P. and Strunski, V., 2003. The inferior laryngeal nerve: surgical and anatomic considerations. Report of 251 thyroidectomies. Surgical and radiologic anatomy, 25(3-4), pp.188-191. https://doi.org/10.1007/s00276-003-0129-7.

[30] Polednak, A.P., 2006. Anatomic configurations of the recurrent laryngeal nerve and inferior thyroid artery with statistical considerations. Surgery, 140(3), pp.481-482. https://doi.org/10.1016/j.surg.2006.02.016.

[31] Beneragama, T. and Serpell, J.W., 2006. Extralaryngeal bifurcation of the recurrent laryngeal nerve: a common variation. ANZ journal of surgery, 76(10), pp.928-931. https://doi.org/10.1111/j.1445-2197.2006.03899.x.

[32] Shao, T., Yang, W., Zhang, T., Wang, Y., Jin, X., Li, Q., Kuang, J., Qiu, W., Chu, P.G. and Yen, Y., 2010. A newly identified variation at the entry of the recurrent laryngeal nerve into the larynx. Journal of investigative surgery, 23(6), pp.314-320. https://doi.org/10.3109/08941939.2010.509465.

[33] Skandalakis, J.E., Droulias, C., Harlaftis, N., Tzinas, S., Gray, S.W. and Akin Jr, J.T., 1976. The recurrent laryngeal nerve. The American surgeon, 42(9), pp.629-634.

[34] Bhargav, P.R.K., 2014. Salient anatomical landmarks of thyroid and their practical significance in thyroid surgery: a pictorial review of thyroid surgical anatomy. Indian journal of surgery, 76(3), pp.207-211. https://doi.org/10.1007/s12262-013-0856-x.

[35] Reed, A.F., 1943. The relations of the inferior laryngeal nerve to the inferior thyroid artery. The Anatomical record, 85(1), pp.17-23. https://doi.org/10.1002/ar.1090850103. 